\title{
dspace.vutbr.cz
}

\section{Prognosis and Health Management in electric drives applications implemented in existing systems with limited data rate}

\author{
KLÍMA, B.; BUCHTA, L.; DOSEDĚL, M.; HAVRÁNEK, Z.; BLAHA, P.
}

2019 24th IEEE International Conference on Emerging Technologies and Factory Automation (ETFA) pp. 870-876

elSBN: 978-1-7281-0303-7

DOI: http://dx.doi.org/10.1109/ETFA.2019.8869520

Accepted manuscript

(C2019 IEEE. Personal use of this material is permitted. Permission from IEEE must be obtained for all other uses, in any current or future media, including reprinting/republishing this material for advertising or promotional purposes, creating new collective works, for resale or redistribution to servers or lists, or reuse of any copyrighted component of this work in other works. KLÍMA, B.; BUCHTA, L.; DOSEDĚL, M.; HAVRÁNEK, Z.; BLAHA, P., "Prognosis and Health Management in electric drives applications implemented in existing systems with limited data rate", 2019 24th IEEE International Conference on Emerging Technologies and Factory Automation (ETFA), pp. 870-876, 2019. DOI: 10.1109/ETFA.2019.8869520. Final version is available at https://ieeexplore.ieee.org/document/8869520 


\title{
Prognosis and Health Management in electric drives applications implemented in existing systems with limited data rate
}

\author{
Klima, B., Buchta, L., Dosedel, M., Havranek, Z., Blaha, P. \\ Central European Institute of Technology, Brno University of Technology \\ Purkynova 123, Brno, Czech Republic \\ \{bohumil.klima, ludek.buchta, martin.dosedel, zdenek.havranek, petr.blaha\}@ceitec.vutbr.cz
}

\begin{abstract}
Importance of the condition monitoring and predictive maintenance in motion systems is growing up as motion systems quantum and their complexity (number of axes, performance parameters) increases with increasing the automation of huge range of human activities and manufacturing processes. Probability of failures increases with the system complexity.

Many faults and indication of their propagation in the electric drives would require additional sensors or hardware, higher bandwidth and sampling frequencies of feedback sensors, high computing power etc. for development of sophisticated methods to detect specific faults with good sensitivity, robustness and reliability under any operating condition.

This paper presents an approach to the condition monitoring and prognosis applicable into the existing systems. These methods use the information available in the traditional electric drives - especially the information from the individual sensors in a voltage source inverter (VSI) and/or an electric motor. Condition indicators for these methods are based on application specific operating states or actions, which generates typical patterns in the signals. The condition monitoring is based on observing the deviations of these patterns between the healthy system and the system with fault propagating. The implementation strategy is described in the paper and some demonstration examples are shown as well.
\end{abstract}

Index Terms-prognosis and health management, PHM, predictive maintenance, electric drive, condition indicator

\section{INTRODUCTION}

Prognosis and health management (PHM) in any technical field is an actual topic in present. The motivation of using them is in a system productivity, reliability and cost effectivity increasing. Modern sensors, communications, computing hardware and theories provide more and more capable resources to implement the reliable solutions for PHM tasks. The effort of the technicians and scientist in this field is presented in many papers [1]-[4], but not many of them concentrate on the implementation strategies into the existing systems.

This work focuses on condition monitoring and predictive maintenance in the existing mechatronic systems. The Fig. 1 defines a general mechatronic system structure regardless if existing or new the system is. The structure can be divided into three layers: Layer 1 - an actuator and sensor layer which includes VSIs and motors with their sensors. The

This work was supported by the H2020 ECSEL Joint Undertaking under grant agreement No. 737453 I-MECH project "Intelligent Motion Control Platform for Smart Mechatronic Systems"
Layer 2 represents centralized real-time controllers like PLCs and Layer 3 is consisted of remote PCs and data storages, parametrizing, watching and logging purposes. Generally, each device in all layers can contain any computing hardware, data storage and is able to communicate its data. The industrial fieldbus communication like EtherCAT is supposed between the Layers 1 and 2 serving for real-time control and data exchange. General ethernet communication is supposed between the Layers 2 and 3.

All functionalities developed under this work need unified strategy of implementation over the structure. The unification rules create a platform [5] which should allow to extend any mechatronic system with functionalities developed in the project. One important rule is that $\mathrm{SW}$ functions are developed under MATLAB Simulink. This enviroment allows translations of Simulink blocks onto functions for various platforms using $\mathrm{C} / \mathrm{C}++$ coder or PLC coder avaliable under Matlab.

PHM contains a chain of the tasks shown in Fig. 2 and described in [6]. The tasks can be distributed over the defined layers in various ways. In the article are presented distributions and implementation approaches to the problem. Quantities and process variables acquisition is always placed into Layer 1. As a good example, quantities measured by the inverter sensors (DC link voltage, heatsink temperature, motor position, phase currents,...) can be considered or the additional sensors can be installed into the Layer 1 (vibration sensors). On the other hand, decision making activities need to be close to operators and should be implemented into Layer 3. Implementation of the other tasks of the PHM over all layers is a question of possibilities of running user programs in the devices on the individual layers and communication load optimization. Some devices (drive inverters) cannot execute user code and data preprocessing is not possible to perform inside them. All data with demanded sampling frequency for diagnosis purposes need to be transferred into upper layers and the communication lines load increases. In case of some preprocessing possibility in Layer 1 condition indicator computation can be performed in this layer. Reduced data are transfered into the upper layers for trend recording, RUL calculation and decission making. Three different approaches to condition indicator obtaing in existing mechatronic system are presented in following. 


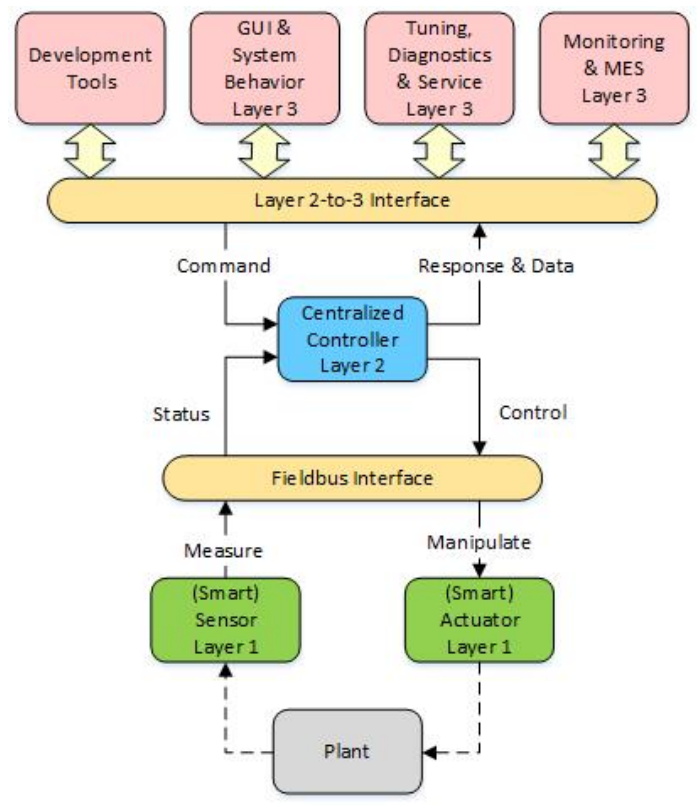

Fig. 1. Mechatronic system structure and layers definition.

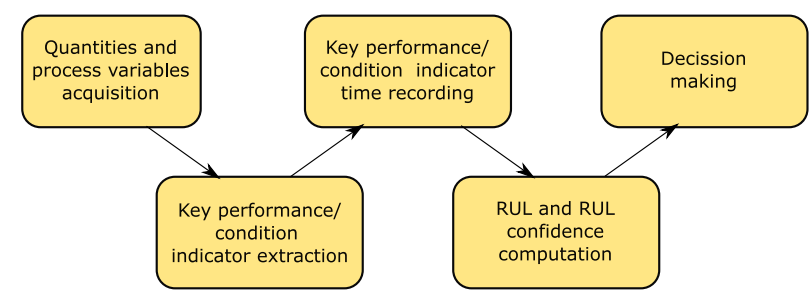

Fig. 2. Prognosis and health management flowchart.

\section{CONDITION INDICATOR IMPLEMENTATION OVER MULTIPLE LAYERS}

Optimization and a good performance of the diagnostic functionalities in the existing system may utilize all resources in the structure according to Fig. 1. Sensors, signal acquisition $\mathrm{HW}$, computing devices and communication lines in individual layers can be assumed like these resourecs. All the resources have to be utilized in the reasonable way to keep the them under exhausting their limits to keep all original functionalities reliable. Especially data preprocessing/condition indicator computation in Fig. 2 can require multiply higher data acquisition rate and shorter computation cycle then the communication interval over all layers is. Significant data reduction can be reached by performing the data preprocessing in the lower layers to utilize computation and communication capability reasonably. Many devices and inverters on Layer 1 can perform user programmed functions beside hard implemented control functionalities. It can be the manufacturer's specific scripting language, some standardized programmable system (e.g. IEC61131-3 PLC) or a user program running under the certain operating system.

\section{A. The $i^{2} t$ condition indicator}

Well known $i^{2} t$ thermal protection method can be adopted for the condition monitoring like a condition indicator in case of monitoring a repeating process. The $i^{2} t$ protection method is typically used in the electric drives for a motor winding burning prevention due to over-current. The difference between square of the instantaneous current and square of nominal current is integrated/summed over the time according to (1). In the other words, the thermal effect of over-current is represented by the $i^{2} t$ value. The bottom limit of the integral is zero.

$$
i^{2} t_{k}=i^{2} t_{(k-1)}+\left(i_{m(k)}^{2}-i_{n o m}^{2}\right) T_{s}
$$

The $i^{2} t$ value is compared to the $i^{2} t_{\text {peak }}$ value which is given for the protected device, for example motor winding. The $i^{2} t_{\text {peak }}$ value is represented by $i_{\text {peak }}$ and $T_{\text {peak }}$ for the most of the protected systems. The threshold value of $i^{2} t$ protection is calculated according to (2)

$$
i^{2} t_{\text {peak }}=\left(i_{\text {peak }}^{2}-i_{\text {nom }}^{2}\right) T_{\text {peak }}
$$

The protection is activated, when $i^{2} t$ value reaches the limiting value $i^{2} t_{\text {peak }}$ and deactivated is after reaching the zero value. The drive torque is limited during protection active state for nominal value typically.

Many mechatronics systems repeat the same action every working cycle under the same operating condition. Repeating motion action with constant mechanical load can be characterized by a typical energy consumption, action time, and repating time courses of many state variables e.g. torque, torque and magnetizing currents and many others. In the other words, the state variables patterns can be compared and analysed with difference expected for healthy and fault propagating system.

$I^{2} t$ calculation according to (1) described above can be adopted for current pattern analysis in such situations. $I_{\text {nom }}^{2}$ parameter value is replaced by the typical current value for repeating action or slightly lower. Bottom limit of the integrator input signal is added and set to zero value assuring upward integration only during repeated cycle.

\section{B. $I^{2} t$ condition indicator simulation}

The simulink scheme of $i^{2} t$ calculation is shown in Fig. 3 . Using is assumed for PMSM drive thus the current inputs are $i_{d}$ and $i_{q}$. Current vector magnitude is calculated based on them. Integrator has to be cleared at the beginning and at read the end of action. This functionality is implemented by two enabled subsystems in Fig. 4. First one contains $i^{2} t$ calculation scheme from Fig. 3. Enable signal allows integration in active state and resets the integrator in inactive state. The second subsystem uses inverted enable signal and serves for holding integral value between falling edges of the enable signal.

The simulation in Fig. 5 shows $i_{d}$ input current to the simulated method in the first graph. The current course represents drive repeating acceleration and deceleration. The magnitude of the current increases each cycle representing increasing 


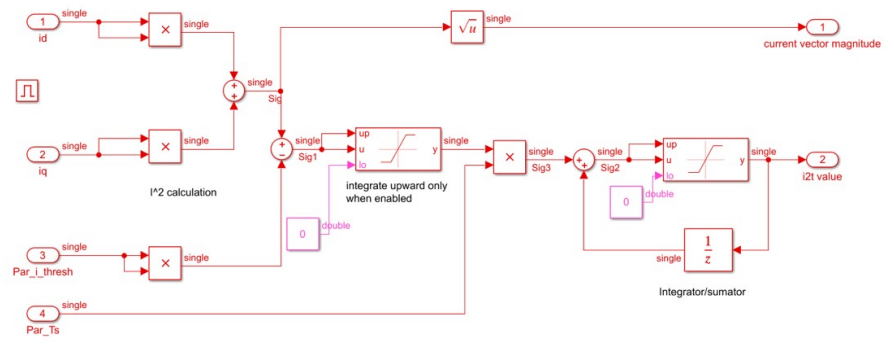

Fig. 3. $I^{2} t$ equation implementation in Simulink.

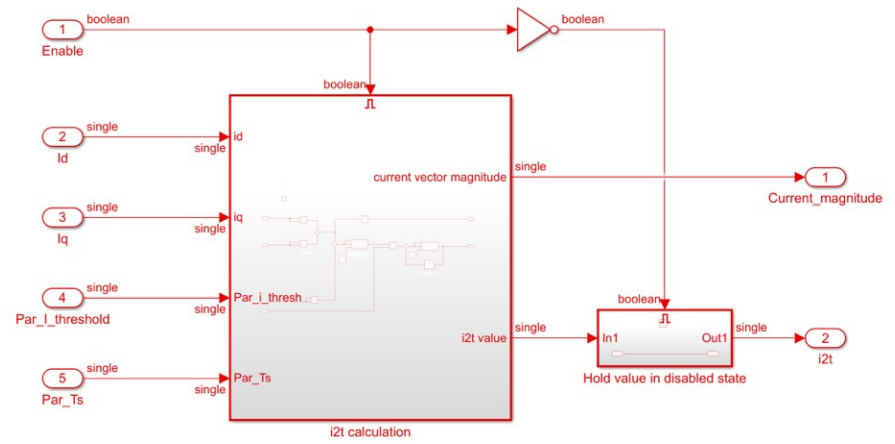

Fig. 4. Enable signal implementation in Simulink for $i^{2} t$ condition indicator.

friction. The increase is $1 \%$ during the whole simulation. The inreasing of $i^{2} t$ indicator value in the second course is $15 \%$. It shows good method sensitivity to detect any friction issues in the drive of permanent magnet weakening and other issues leading to increasing current magnitude for any repeating actions.

\section{Experimental implementation}

The experimental verification shows usage of the algorithm developed in Simulink. Algorithm was translated using MATLAB PLC coder and compiled in Gefran ADV200-S commercial inverter. The inverter allows running Structured Text PLC programs according to IEC 61131-3. Inverter was connected to PMSM that was coupled with dynamometer. Test setup is shown in Fig. 6. Fig. 7 shows the condition
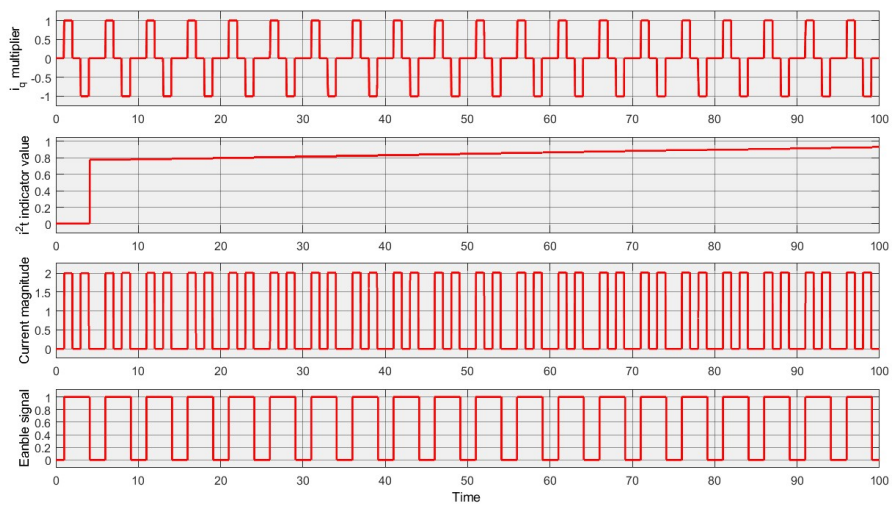

Fig. 5. $i^{2} t$ condition indicator simulation results.

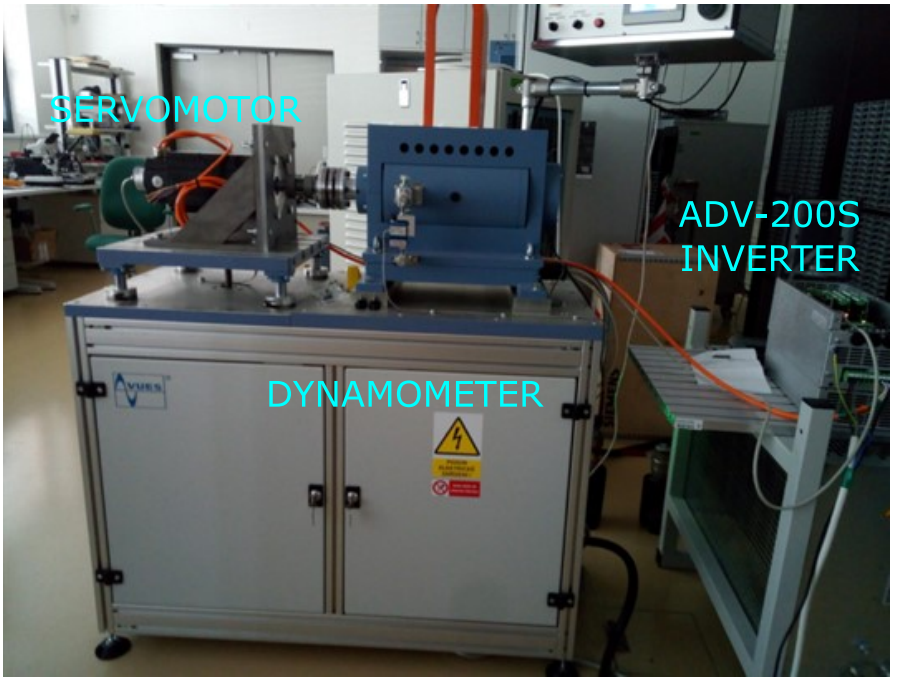

Fig. 6. Laboratory setup for verification.

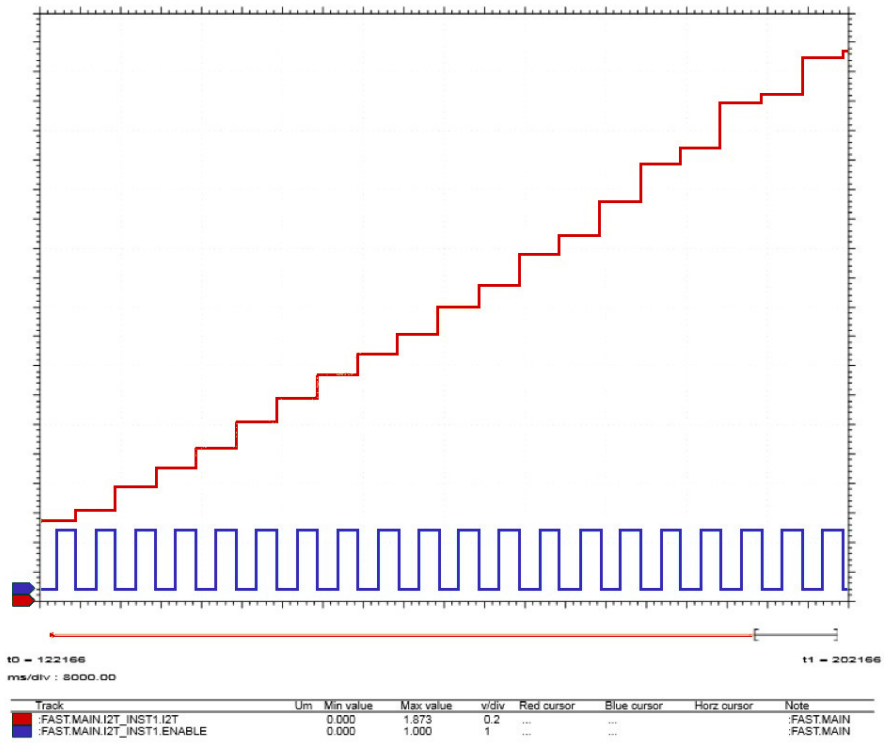

Fig. 7. $i^{2} t$ condition indicator lab verification.

indicator value (red line) and the enable signal (blue line). The torque on the shaft was increasing and the value of the indicator rises as expected. The dynamometer weren't able to generate alternating load torque like in simulation is presented. Implementation correctness was checked and proven by manual calculation according to (1). Functionality of the enable signal is proven as well.

\section{CONDITION INDICATOR IMPLEMENTATION IN THE LAYER 3}

The condition monitoring of electrical drive systems is very often based on the spectral analysis of the measured quantities. In the case of electric drives, the currents, voltages, velocities and positions are the most frequently measured quantities. The spectral analysis is most often carried out from measured 
phase currents in practice [7], [8]. Typically, this method requires data that are sampled at the same or even higher sampling frequency as the frequency of PWM update, which corresponds to measurement on the Layer 1. Unfortunately, data with the required sampling rate are usually not available on the Layer 3. It is assumed that measured process quantities will be available on the Layer 3 at $500 \mathrm{~Hz}$ sampling frequency. This sampling frequency is insufficient for accurate spectral analysis of the measured quantities of the electrical drives. It is appropriate to use different approach that is able to operate with measured data at this sampling rate, due to the above mention limitation.

\section{A. Park's vector approach}

In practice, the Park's vector approach is used to detect an inter-turn short-circuit [9]-[11], open-phase fault or all phase open. This approach is also used to diagnose the state of the rotor cage of asynchronous motors [12]. A broken rotor bar can be detected by deformation of the circle shape of the Park's vector pattern [13], [14].

The Park's vector method is based on the analysis of current complex vectors of the three-phase electric motor in $\alpha \beta$ coordinates [15]. There are three space vectors $(a, b, c)$ for three-phase motor that are mutually shifted by $120^{\circ}$. The analysis of the three-phase motor can be simplified using the Clark transformation. This transformation allows reducing of a three-phase system into a two-phase equivalent system.

$$
\left[\begin{array}{c}
i_{\alpha} \\
i_{\beta}
\end{array}\right]=\left[\begin{array}{rrr}
\frac{2}{3} & -\frac{1}{3} & -\frac{1}{3} \\
0 & \frac{1}{\sqrt{3}} & -\frac{1}{3}
\end{array}\right]\left[\begin{array}{c}
i_{a} \\
i_{b} \\
i_{c}
\end{array}\right]
$$

Where $i_{\alpha}$ and $i_{\beta}$ represent components of the Park's complex current vector. Subsequently, the individual complex vectors can be displayed in the $\alpha \beta$-coordinate system with respect to electrical position $\theta_{e}$.

Assuming that the phase currents were measured in a steady state at least during one electrical revolution, it is possible to create a circular pattern centered on the origin of the $\alpha \beta$ - coordinate system. The Park's current curve should be a circular shape in the case of a healthy motor and gearbox. In the case of faulty motor or gearbox, the shape of the Park's current curve is changed. The circular shape can be deformed by parasitic harmonic components which are occurred in phase currents. Therefore, the basis of the motor health evaluation is the monitoring of the deviation in the current Park's vector pattern from its ideal circular shape. The deviation increases with severity of the faults in motor or gearbox.

The measurements of the phase currents should be performed in steady state under known operating conditions. In the real case, it may happen that it is not possible to measure simultaneously a complete data set to obtain the current Park's vector pattern under defined operation condition. Further complication may arise if the sampling rate is not high enough for the Park's vector pattern to be composed of a sufficient number of samples for condition monitoring. The data preprocessing

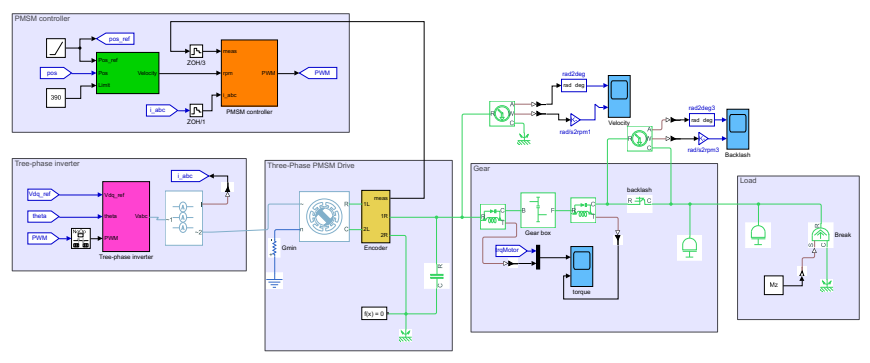

Fig. 8. Verification of the Park's vector approach.

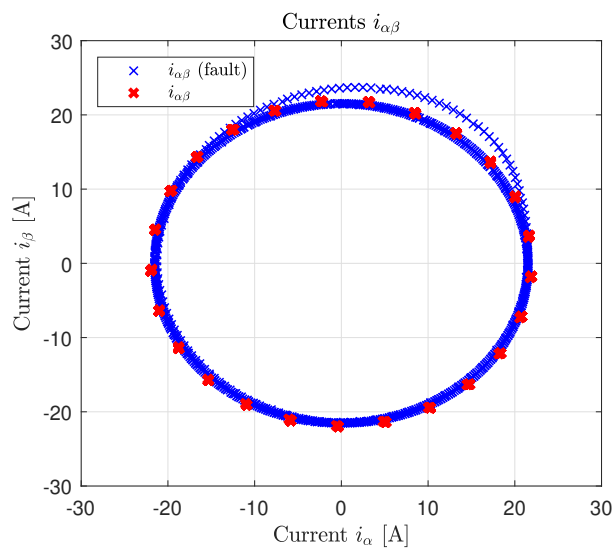

Fig. 9. The current Park's vector patterns.

should be done if mentioned situations happen. The data preprocessing can be used to find sections that were measured under the same operation conditions but at different times. Subsequently, the resulting current Park's vector pattern can be composed of several parts depending on the rotor position.

\section{B. Simulation experiments}

The verification of the Park's vector approach is carried out on the model of the drive system in the MATLAB/Simulink 2018b. Simulink block diagram (Fig. 8) is composed of the inverter model, PMSM model, vector control algorithm and mechanical part of the system. The gearbox model includes meshing and viscous losses and fault model which allows to set the rotational angle range for the faulted efficiency. Furthermore, the backlash in gearbox is implemented which it is defined by the difference between the position of the motor shaft and the position of the output shaft of gearbox.

This subsection presents the simulation results of the Park's vector approach. Fig. 9 shows the resulting current Park's vector patterns for two states of the drive system that were obtained with respect to the same operation condition. Shown patterns are obtained from data that were measured in the steady state (constant rotor speed). The data were measured at the frequency of $500 \mathrm{~Hz}$. The first current Park's vector pattern (blue crosses) represents the behavior of the drive system when the efficiency of the gearbox is set to $5 \%$ in the rotational angle range $5^{\circ}$. The fault is reflected by 


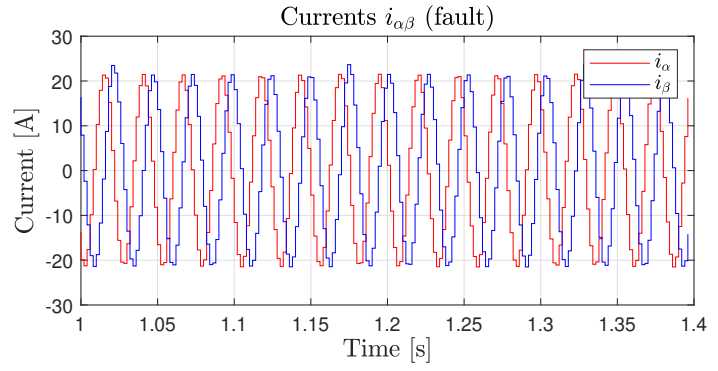

Fig. 10. The $\alpha \beta$-currents of PMSM with bearing fault.

circular shape deformation in the part of the pattern range that corresponds to one electric motor revolution. For a more detailed demonstration of the fault state of drive system, the pattern is composed of data that was measured during more mechanical revolutions. There is also shown pattern which presents state of the drive system without fault status (red crosses) for the comparison.

The $\alpha \beta$-current waveforms in time domain are shown in Fig. 10. The error status is almost impossible to detect in $\alpha \beta$ current waveforms in time domain when currents are measured at sampling frequency $500 \mathrm{~Hz}$. In contrast, the comparison of current Park's vector patterns from the Fig. 9 confirms the fault.

\section{ADDITIONAL SENSOR INSTALLATION}

\section{A. The diagnostics based on a vibration signal}

The vibration based diagnostics brings a significant advantage in the overall diagnostics of the machines [16]. It has a strong contribution to the complete probability of the evaluation of the propulsion health.

\section{B. Diagnostics approaches}

Several approaches of the diagnostics can be performed. First type, well known and commonly used in the industrial applications, contains of a vibration sensor mounted on the measured machine and an evaluating front-end equipment. All the data are transferred to the supervising system for postprocessing and evaluation. A lot of measuring places are processed simultaneously by almost one measuring device. A significant part of the intelligence is implemented in the upper system layer. Second type of the diagnostics approach lies in using of some smart sensors performing a simple signal processing and providing a decision system (upper layers) only with the simple KPIs describing the overall status of the machine or RUL estimation. A third type combines the two aforementioned possibilities in a different ratio.

\section{Key parameters in vibrodiagnostics}

The RMS value in ISO band is very common and useful value in the vibrodiagnostics [17]. Almost all the machines can be evaluated according to this value. It has to be mentioned, that not the absolute value of the vibration signal is a key parameter, but its relative change compared to the initial state. Thus it is important to store the historical values and create a trend of the measured quantity. Since the RMS value is an overall pointer of the machine health, its increased value only serves as an indicator that something is happening with the machine. However the type of the failure cannot be identified from this value. A frequency analysis is a good procedure for a detailed diagnostics of the machine, since every machine failure has its response in the frequency spectrum.

\section{Smart vibration sensor (SVS) performance}

A complete vibration sensor consisting of two MEMS sensors has been developed - see Fig. 11. Its advantage

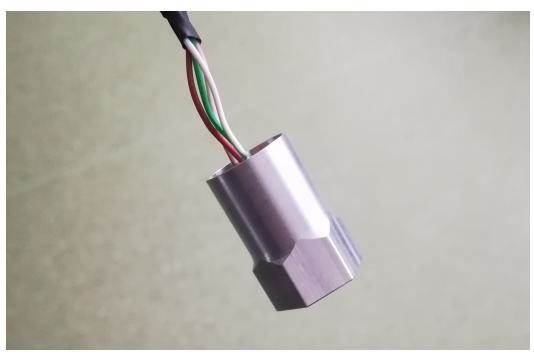

Fig. 11. A photography of the SVS.

compared to the standard industrial ICP sensor [18] is in an extended temperature range (up to $125{ }^{\circ} \mathrm{C}$ ), a lower price (at a tenth of the price for the standard accelerometer), an increased reliability (due to using of two sensing elements) and a modularity (the function of the sensor is strongly defined by an internal firmware). Sensor measures signal from the two accelerometers (the primary type, ADXL1002, is an uniaxial with a HF range of up to $11 \mathrm{kHz}$ and the secondary, ADXL355, is a triaxial type with a LF range of up to $1 \mathrm{kHz}$ ) and transfers it through RS485 bus to the upper supervising system. There is currently no additional signal processing inside the sensor due to relatively strict requirements for the complete timing of the system and a low performance of the used processor. A more detailed description can be found in [19]. The simplified schematics of the sensor can be seen in the Fig. 12. Key param-

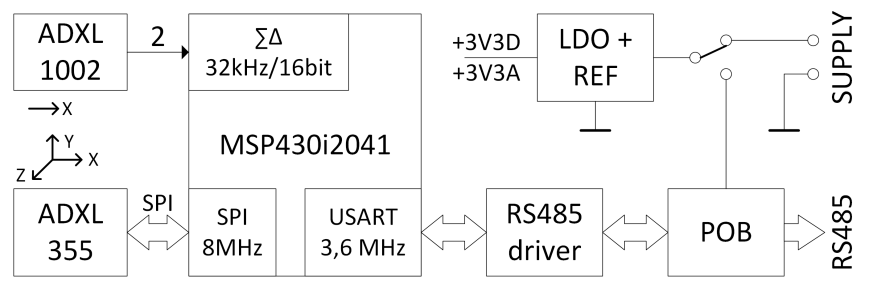

Fig. 12. A block schematics of the SVS.

eters of the sensor were measured on the calibration system SPEKTRA CS18 with very good results - mainly sensitivities of the particular elements, their frequency responses and cross axial sensitivities. Measured frequency response of the sensor can be seen in the Fig. 13. The characteristic is almost ideal for both sensing elements, without any resonance. This results confirm a good performance of the sensor for the industrial 


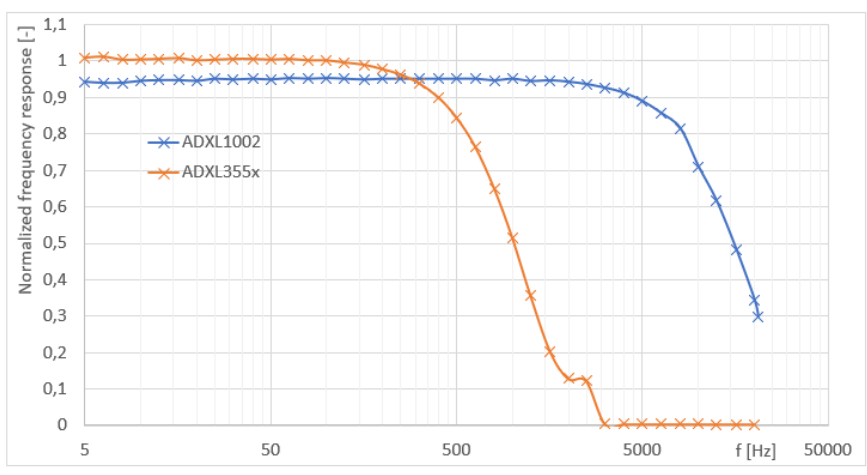

Fig. 13. Frequency response of the SVS.

measurement applications in ISO band as well as other high frequency measurements.

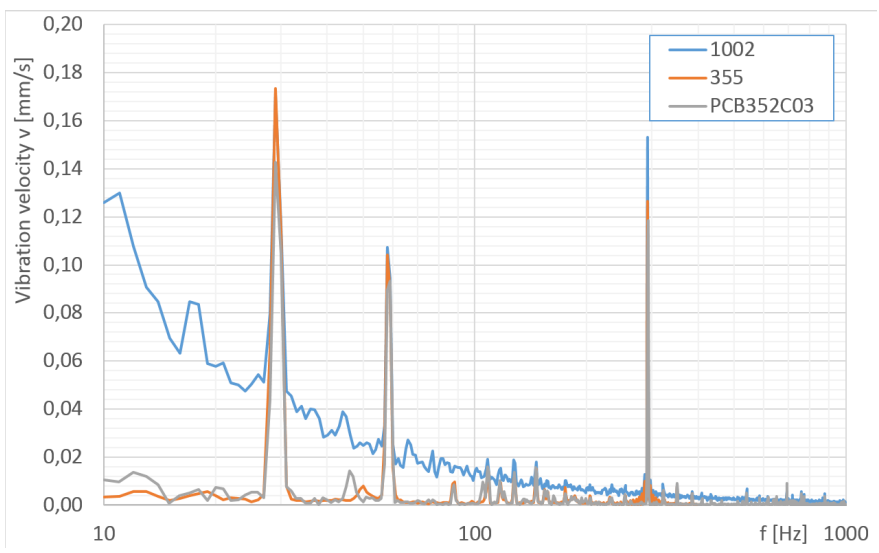

Fig. 14. The frequency spectrum of the damaged motor measured by the SVS.

Also some practical issues were measured by the sensor due to confirm its ability of measuring in the industrial applications. A simple motor and actuator were used as an industrial machines with a different faults (unbalance, damaged bearing) and the vibration signal was measured by the sensor. Measured spectrum of the unbalanced 10-pole motor with the bent shaft can be seen in the Fig. 14. First harmonic component represents unbalance of a rotor, second harmonic represents bent shaft and the last harmonic $\left(10^{\text {th }}\right)$ represents number of poles in the rotor.

\section{E. Resume}

A complex measurement system for vibration diagnostics containing the SVS and a supervising application (Ni cRIO, LabVIEW application) has been developed and its parameters in both the laboratory and the industrial environments were checked with promising results.

\section{CONCLUSION}

Implementation of the predictive maintenance functionalities into existing mechatronic system is demanded subject to increase their effective utilization and minimize downtimes.
The purpose of the article was finding ways how to implement PHM functionalities into legacy mechatronic systems. Structure and layers of general industrial mechatronic system were defined. Ways of condition indicators implementation were presented in the paper like most critical functionalities in point of view of implementation techniques and data flow optimization over defined layers. The other PHM functionalities like condition indicators recording and RUL estimation are supposed purely in Layer 3. Sufficient computing performance and data storage capability is assumed in Layer 3.

Three approaches of these functionalities realizations differing in over-layers implementation were presented in the article. The methods are based on existing sensors data and process variables of the drive or on adding external sensors as well.

The first presented approach advantage is low data flow between individual layers. Condition indicators implemented in lower layers significantly reduce data flow for PHM purposes. However, the method requires low layer devices with capability of executing user defined functions. The method cannot be universal for all brownfield devices and requires various ways of programs downloading, parametrizing and configuring.

The second way is implementation of condition indicators into Layer 3. This approach is implementable much more universally but requires transferring of all necessary process data to Layer 3. The data sampling frequency is usually low due to limited available capacity of communication lines. Condition indicators need to by adopted for low sampling frequencies for this case. The averaged Park's vector trajectory has to be acquired for multiple turns of motor and can be assumed like a suitable method for Layer 3. Various parts degradations and faults can be evaluated from shape of the Park's Vector Trajectory.

The third approach is fully universal, because dedicated hardware is used. This solution can be implemented into both the greenfield and brownfield. Mechanical vibrations are measured by the Smart Vibrations Sensor, which is considered as Layer 1 device, processed and handover to the upper layers as the condition indicators. This approach does not set any requirements for the system topology or inter-layers communication capabilities since all the processing is done in the separated hardware resource. This approach can be applied also for the above mentioned ways - to sense e.g. phase currents of the motor by a non-contact sensors (e.g. Hall probes) and using a dedicated high performance and high sampling speed capabilities hardware to compute the condition indicators itself and only transfer them to the upper layers for further processing.

\section{REFERENCES}

[1] E. Strangas and S. Aviyente, "Failure prognosis methods in electrical drives - State of the art and future directions," in 2015 IEEE Workshop on Electrical Machines Design, Control and Diagnosis (WEMDCD). IEEE, mar 2015, pp. 254-260. [Online]. Available: http://ieeexplore.ieee.org/document/7194538/ 
[2] Bin Lu and S. Sharma, "A Literature Review of IGBT Fault Diagnostic and Protection Methods for Power Inverters," IEEE Transactions on Industry Applications, vol. 45, no. 5, pp. 1770-1777, 2009. [Online]. Available: http://ieeexplore.ieee.org/document/5165060/

[3] M. C. Garcia, M. A. Sanz-Bobi, and J. del Pico, "SIMAP: Intelligent System for Predictive Maintenance," Computers in Industry, vol. 57, no. 6, pp. 552-568, aug 2006. [Online]. Available: https://linkinghub.elsevier.com/retrieve/pii/S0166361506000534

[4] A. K. Jardine, D. Lin, and D. Banjevic, "A review on machinery diagnostics and prognostics implementing conditionbased maintenance," Mechanical Systems and Signal Processing, vol. 20, no. 7, pp. 1483-1510, oct 2006. [Online]. Available: https://www.sciencedirect.com/science/article/pii/S0888327005001512

[5] M. Cech, A.-J. Beltman, and K. Ozols, "I-MECH - Smart System Integration for Mechatronic Applications," in IEEE ETFA 2019. Zaragoza, Spain: Paper in Press, 2019.

[6] Y. Lei, N. Li, L. Guo, N. Li, T. Yan, and J. Lin, "Machinery health prognostics: A systematic review from data acquisition to RUL prediction," Mechanical Systems and Signal Processing, vol. 104, pp. 799-834, 2018.

[7] L. Romeral, J. C. Urresty, J.-R. Riba Ruiz, and A. Garcia Espinosa, "Modeling of Surface-Mounted Permanent Magnet Synchronous Motors With Stator Winding Interturn Faults," IEEE Transactions on Industrial Electronics, vol. 58, no. 5, pp. 1576-1585, may 2011

[8] W. le Roux, R. G. Harley, and T. G. Habetler, "Detecting Rotor Faults in Low Power Permanent Magnet Synchronous Machines," IEEE Transactions on Power Electronics, vol. 22, no. 1, pp. 322-328, jan 2007.

[9] A. Marques Cardoso, S. Cruz, and D. Fonseca, "Inter-turn stator winding fault diagnosis in three-phase induction motors, by Park's vector approach," IEEE Transactions on Energy Conversion, vol. 14, no. 3, pp. 595-598, 1999.

[10] L. M. R. Oliveira and A. J. M. Cardoso, "Intermittent turn-to-turn winding faults diagnosis in power transformers by the on-load exciting current Park's Vector Approach," in 2008 18th International Conference on Electrical Machines. IEEE, sep 2008, pp. 1-6.

[11] T. G. Vilhekar, M. S. Ballal, and H. M. Suryawanshi, "Application of double Park's vector approach for detection of inter-turn fault in induction motor," in 2015 International Conference on Condition Assessment Techniques in Electrical Systems (CATCON). IEEE, dec 2015, pp. $173-178$.

[12] N. Benouzza, A. Benyettou, and A. Bendiabdellah, "An advanced park's vectors approach for rotor cage diagnosis," in First International Symposium on Control, Communications and Signal Processing, 2004. IEEE, 2004, pp. 461-464.

[13] Y. Soufi, T. Bahi, S. Lekhchine, H. Merabet, and M. F. Harkat, "Broken bars fault diagnosis in induction machine fed by a voltage source inverter," in 2013 Eighth International Conference and Exhibition on Ecological Vehicles and Renewable Energies (EVER). IEEE, mar 2013, pp. 1-6.

[14] T. Vaimann, A. Belahcen, J. Martinez, and A. Kilk, "Detection of broken bars in frequency converter fed induction motor using Park's vector approach," in 2012 Electric Power Quality and Supply Reliability. IEEE, jun 2012, pp. 1-4.

[15] D. Diallo, M. Benbouzid, D. Hamad, and X. Pierre, "Fault Detection and Diagnosis in an Induction Machine Drive: A Pattern Recognition Approach Based on Concordia Stator Mean Current Vector," IEEE Transactions on Energy Conversion, vol. 20, no. 3, pp. 512-519, sep 2005.

[16] P. Girdhar and C. C. Scheffer, Practical machinery vibration analysis and predictive maintenance. Elsevier, 2004.

[17] P. E. Mix, Introduction to nondestructive testing : a training guide. Wiley, 2005.

[18] E. Spence, "What You Need to Know About MEMS Accelerometers for Condition Monitoring - Analog Devices," 2016. [Online]. Available: https://www.analog.com/en/technicalarticles/mems-accelerometers-for-condition-monitoring.html

[19] M. Doseděl and Z. Havránek, "Design and performance evaluation of smart vibration sensor for industrial applications with builtin MEMS accelerometers - IEEE Conference Publication," in 2018 18th International Conference on Mechatronics Mechatronika (ME). Brno: IEEE, 2018. [Online]. Available: https://ieeexplore.ieee.org/document/8624720 